University of Nebraska - Lincoln

DigitalCommons@University of Nebraska - Lincoln

8-2005

\title{
Stochastic Modeling of the Permeability of Randomly Generated Porous Media
}

\author{
Yusong Li \\ University of Nebraska - Lincoln, yli7@unl.edu \\ Eugene LeBoeuf \\ Department of Civil and Environmental Engineering, Vanderbilt University, Nashville, TN \\ P. K. Basu \\ Department of Civil and Environmental Engineering, Vanderbilt University, Nashville, TN \\ Sankaran Mahadevan \\ Department of Civil and Environmental Engineering, Vanderbilt University, Nashville, TN
}

Follow this and additional works at: https://digitalcommons.unl.edu/civilengfacpub

Part of the Civil Engineering Commons

Li, Yusong; LeBoeuf, Eugene; Basu, P. K.; and Mahadevan, Sankaran, "Stochastic Modeling of the Permeability of Randomly Generated Porous Media" (2005). Civil Engineering Faculty Publications. 8. https://digitalcommons.unl.edu/civilengfacpub/8

This Article is brought to you for free and open access by the Civil Engineering at DigitalCommons@University of Nebraska - Lincoln. It has been accepted for inclusion in Civil Engineering Faculty Publications by an authorized administrator of DigitalCommons@University of Nebraska - Lincoln. 
Published in Advances in Water Resources 28: 8 (August 2005), pp. 835-844; doi 10.1016/j.advwatres.2005.01.007

Copyright (C) 2005 Elsevier Ltd. Used by permission. http://www.sciencedirect.com/science/journal/03091708

Submitted October 11, 2004; revised January 27, 2005; accepted January 28, 2005; published online March 28, 2005.

\title{
Stochastic Modeling of the Permeability of Randomly Generated Porous Media
}

\author{
Yusong Li, Eugene J. LeBoeuf, P.K. Basu, and Sankaran Mahadevan \\ Department of Civil and Environmental Engineering, Vanderbilt University, Nashville, TN 37325, USA \\ Corresponding author - E. J. LeBoeuf, tel 615 343-7070, fax 615 322-3365, email eugene.j.leboeuf@vanderbilt.edu
}

\begin{abstract}
Permeability of porous media in subsurface environments is subject to potentially large uncertainties due to the heterogeneity of natural systems. In this study, a first-order reliability method (FORM) is combined with a lattice Boltzmann method (LBM) to estimate the permeability of randomly generated porous media. The proposed procedure provides an increased ease of addressing complex pore structures by employing LBM to model fluid flow, while inheriting the computational efficiency from FORM. Macroscale-equivalent permeability can thus be estimated with significantly reduced computational efforts, while maintaining a connection to the complex microscale fluid dynamics within a pore structure environment. Implemented on several randomly generated porous media domains, the proposed method provides 13-120 times the efficiency compared to Monte Carlo methods.
\end{abstract}

Keywords: permeability, porous media, lattice Boltzmann method, first order reliability method, stochastic modeling

\section{Introduction}

It is well recognized that fluid flow in subsurface porous media is strongly influenced by spatial variability and heterogeneity [1]. Proper modeling of groundwater flow within this environment thus involves capture of multiscale phenomena, including microscale- (molecular), mesoscale- (single pore), macroscale- (multiple pores), and megascale- (field) level systems. A central challenge arising from this situation is to understand how macroscale characteristics of fluid flow depend on microscale geometry of pore spaces and physical characteristics of the fluid and solid [2].

Specific permeability is an important macroscale parameter representing averaged microscale characteristics of fluids and porous media. At the macroscale, specific permeability for single-phase flow can be described within the context of Darcy's law for low Reynolds numbers:

$$
q=-\frac{k}{\mu}(\nabla p+\rho g \nabla z)
$$

where $k\left[\mathrm{~L}^{2}\right]$ is the specific permeability, $q[\mathrm{~L} / \mathrm{T}]$ is the specific flow rate, $\mu[\mathrm{M} / \mathrm{LT}]$ is the viscosity of the fluid, $\nabla p\left[\mathrm{M} / \mathrm{L}^{2} \mathrm{~T}^{2}\right]$ is the pressure gradient, $\rho$ is the fluid density $\left[\mathrm{M} / \mathrm{L}^{3}\right], g$ is the gravitational acceleration $\left[\mathrm{L} / \mathrm{T}^{2}\right]$, and $z[\mathrm{~L}]$ is the vertical coordinate. Furthermore, many empirical methods, such as the Hazen method and Kozeny theory [1], have related specific permeability with microscale properties of porous media, including particle size, sorting level, and porosity. For example, the Kozeny equation may be expressed as:

$$
k=\frac{\phi^{3}}{c S^{2}}
$$

where $\phi[-]$ is the porosity, $c[-]$ is the Kozeny coefficient, and $S[1 / \mathrm{L}]$ is the specific surface area defined by the 
ratio of particle surface area exposed to fluid per unit volume.

The heterogeneous nature of soils derived in part from the randomness of particle size distributions, porosities, and pore structures, however, suggests that soil and sediment permeability is also subject to randomness and uncertainty. This uncertainty is well recognized, and numerous studies have employed stochastic methods to model groundwater flow in subsurface porous media by assuming a permeability probability density function, including the use of normal [4], lognormal $[3,5,6]$, and gamma $[7,8]$ distributions. Although it is widely understood that the selection of a particular probability density function will markedly influence simulation results [8, 9], few studies [9-11] describe the manner in which to construct a permeability probability density function. These studies primarily focus on experimental determination of probability density functions for permeability at the field scale. Developing numerically-dervied distributions will be more economically efficient, although such efforts face several technical challenges, namely: (i) restriction on the computational resources available to employ numerous Monte-Carlo-type statistical simulations and (ii) difficulty in accurately capturing influences of microscale uncertainties on macroscale permeability uncertainty.

Recently, several analytical reliability approximation methods, e.g., first-order reliability method (FORM) and second-order reliability method (SORM), have been used in the environmental field to model groundwater flow and contaminant transport $[4,12,13]$, and surface water quality [14, 15]. Possessing greater efficiency than traditional Monte-Carlo-type simulations, these methods can greatly decrease computational demands. In addition, lattice Boltzmann methods (LBM) have been applied to estimate the permeability of porous media [16-20]. Due to its ability to address fluid flow in complex micropore geometries, researchers have used LBM to help to relate microscale uncertainties with macroscale permeability uncertainty. In this paper, we present a mathematical framework to construct a probability density function for permeability that (i) employs LBM to estimate permeability based on fluid flow in complex micropore geometries, and (ii) utilizes FORM to derive the stochastic characteristics of porous media permeability. In this way, probability density functions for permeability can be constructed with reasonable computational efforts based on more easily obtained media properties, e.g., porosity and particle size distribution. Although permeability CDFs constructed by LBM FORM in this study focus on the pore scale, this effort has the potential to provide valuable information for correctly constructing permeability CDFs at the field scale [21].
Descriptions of numerical methods employed in this work follow this introduction. This discussion includes a brief introduction to porous media generation methods, the theoretical basis for use of LBM to model fluid flow, and the statistical basis for FORM, concluding with a general description of the proposed algorithm. The subsequent section exemplifies implementation of LBM FORM in several example domains of interest, including discussion of statistical properties of the generated permeability density function, and the accuracy and efficiency of the new method. Following a brief summary, the manuscript concludes by highlighting directions for further enhancements of the proposed method.

\section{Numerical methods}

\subsection{Porous media generation}

Accurate numerical simulation of fluid flow in porous media requires detailed descriptions of porous media morphology, which should include geometric properties such as particle or pore shape and volume, and topological properties such as pore interconnectivity. In many cases, however, the type of model that can be employed is dependent on the modeling method, and more importantly, limited computational resources. It is thus important to construct models that are able to closely mimic the heterogeneity of actual porous media, and at the same time are sufficiently efficient to allow simulation of flow and transport phenomena with reasonable computational effort. In this study, porous media are envisioned as a statistical distribution of non-overlapping circular disks representing soil particles distributed in a rectangular two-dimensional uniform continuum representing the pore space through which a fluid flows.

As first proposed by Gardner, particle size distributions in soil are often assumed to be lognormal in nature [22]. Buchan noted that approximately one-half of the US Department of Agriculture (USDA) textual classification triangle could be adequately modeled by a lognormal distribution. Since a standard lognormal distribution implies zero and infinity for the smallest and largest particle sizes, respectively, modified lognormal distributions were developed to constrain the upper and lower extremes of the particle size. Recently, Fredlund et al. [23] proposed a new model based on a unimodal mathematical function, which is believed to provide improved representations of particle size distributions relative to lognormal distributions. This model's ease of use, however, is limited by its employment of five fitting parameters; our study thus employs a modified lognormal distribution to describe particle size distribution, assuming that all particle sizes reside in a $95 \%$ confidence interval to eliminate extremely large or small particles. 
Modifying the algorithm proposed by Yang et al. [24] for a three dimensional sphere packing, a two-step collective rearrangement technique is developed to generate random porous media. First, particles with size distributions following a modified lognormal distribution are generated until the required porosity is satisfied. The particles are then assigned to a two dimensional domain by assuming a uniform distribution of particle locations. Based on this initial, possibly overlapped configuration (i.e., one particle may overlap another particle), an iterative arrangement process is applied to achieve an overlap-free condition. During each iteration, the largest particle is selected for relocation if there is any overlap with another particle; if overlap occurs, its spatial location is adjusted until the overlap is removed, and then registered in the final non-overlap location. The procedure continues with the next largest particle, etc. until all particles are registered in their final non-overlap location. Periodic boundary conditions are maintained at all boundaries throughout the iteration process. Figure 1 provides an illustration of several of the generated random porous media employed in this study.

\subsection{LBM simulation}

LBM $[16,25,26]$ is a mesoscopic approach for simulating computational fluid dynamics by solving a discretized Boltzmann equation. An attractive feature of LBM is the ease of addressing complex boundary conditions by implementing very simple schemes. Numerous works have successfully applied LBM in modeling fluid flow in porous media and quantification of porous media permeability $[16,17,27,28]$.

LBM models fluids as particle distributions residing on a discrete lattice, propagating to their adjacent lattice nodes, and colliding with other particles to redistribute momentum. In this study, a two-dimensional, nine-velocity lattice model (D2Q9) [26] is employed. The evolution process can be expressed by the equation:

$$
f_{i}\left(\vec{x}+\vec{c}_{i}, t+1\right)=f_{i}(\vec{x}, t)+\frac{1}{\tau}\left[f_{i}^{\mathrm{eq}}(\vec{x}, t)-f_{i}(\vec{x}, t)\right]
$$

where $f_{i}$ represents the particle distribution in position $\vec{x}$ at time $t$, moving with velocity $\vec{c}_{i}$, $\tau$ is the relaxation time which controls the rate of approach towards equilibrium, and $f_{i}^{\mathrm{eq}}$ is an equilibrium distribution parameter. It has been shown [29] that the Navier-Stokes equation can be recovered from this discretized Boltzmann equation for incompressible flow, with a truncation error proportional to the square of the Mach number $\left(M_{a}=u / c_{s^{\prime}}\right.$ where $u$ is the characteristic flow velocity, and $c_{\mathrm{s}}$ is the speed of sound (usually set to $1 / \sqrt{3}$ for the D2Q9 model)). The density per node, $\rho$, the macroscopic velocity, $\vec{u}$, the fluid pressure $P$, and the kinematic viscosity $v$ are defined by

$\rho=\sum_{i} f_{i} ; \quad \rho \vec{u}=\sum_{i} f_{i} c_{i} ; \quad P=\rho c_{\mathrm{s}}^{2} ; \quad v=c_{\mathrm{s}}^{2}\left(\tau-\frac{1}{2}\right)$

A non-slip boundary condition is imposed at the solid and liquid interfaces by implementing a bounceback rule that reverses the momentum of particles approaching the solid wall. Periodic boundary conditions are maintained at the inlet and outlet of the domain. A pressure gradient is imposed by maintaining a density difference between the inlet and the outlet of the simulation domain; thus at very small Reynolds numbers, the permeability of simulated porous domains can be estimated by Darcy's law based on the imposed pressure gradient and specific flow rate derived from LBM simulation.

\subsection{FORM}

FORM originated from reliability analysis in structural engineering, and is an attractive alternative to computationally intensive Monte Carlo methods [4]. In this paper, we implement FORM to construct permeability cumulative distribution functions (CDFs) for randomly generated porous media. A description of the FORM procedure is presented below.

In reliability analysis, a function $M\left(x_{1}, x_{2}, \ldots, x_{n}\right)$ is often formulated to describe the performance of a system. The system performance is considered in terms of two

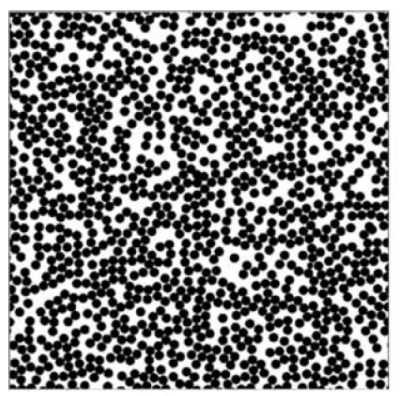

(a)

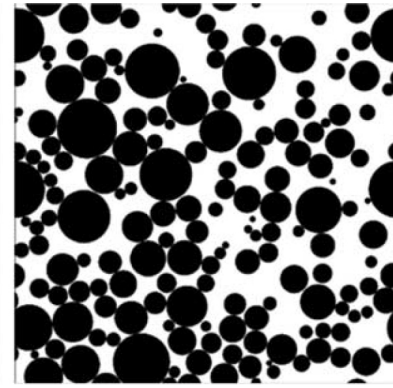

(b)

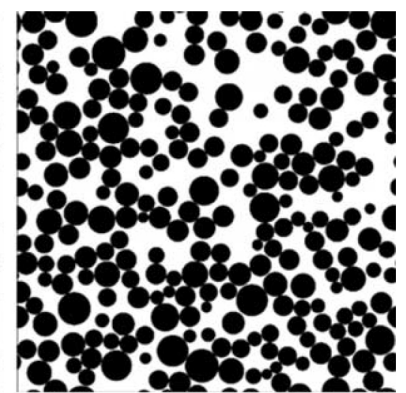

(c)

Figure 1. Example randomly generated porous media at porosity 0.45 : (a) geometric mean diameter is $25 \mu \mathrm{m}$ and coefficient of variation (COV) 0.01; (b) geometric mean diameter is $50 \mu \mathrm{m}$ and COV 0.6; (c) geometric mean diameter is $50 \mu \mathrm{m}$ and COV 0.3 . 
states, 'failure' or 'safe', depending on whether the performance function is less than or greater than zero, respectively. A limit state surface may then be defined as the boundary between the failure and safe regions, i.e., $M\left(x_{1}, x_{2}, \ldots, x_{n}\right)=0$. In this study, the performance function evaluates whether the calculated permeability of a simulation domain is smaller than some selected target value $g_{i}$ :

$$
M\left(x_{1}, x_{2}, \ldots, x_{n}\right)=G\left(x_{1}, x_{2}, \ldots, x_{n}\right)-g_{i}
$$

The limit state surface may thus be defined as $G=g_{i}$. The probability of failure, i.e., $G$ is less than $g_{i}$, can be defined:

$$
\begin{aligned}
F_{G}(g) & =p\left(G \leqslant g_{i}\right) \\
& =\int \ldots \int_{G \leqslant g} f_{X}\left(x_{1}, x_{2}, \ldots, x_{n}\right) \mathrm{d} x_{1} \mathrm{~d} x_{2} \mathrm{~d} x_{n}
\end{aligned}
$$

where $f_{X}\left(x_{1}, x_{2}, \ldots, x_{n}\right)$ is the joint probability density function for random variables $x_{1}, x_{2}, \ldots, x_{n}$. Equation (6) is difficult to evaluate for many reasons, including (i) difficulty of evaluating multidimensional integration; (ii) lack of statistical information for the joint probability density function, $f$; and (iii) the complexity in evaluating the performance function. The objective of FORM is to derive an estimation of $F_{G}$ based on a first-order Taylor series expansion of the performance function.

If random variables, $x_{i}$ s, are correlated non-normal functions, they should be transformed to the space of uncorrelated reduced normal functions [30]. On the transformed limit state surface, the point closest to the origin is defined as the "design point," representing the most likely failure point. This minimum distance from the origin in the transformed space can be computed as

$$
\beta=-\vec{\alpha}^{\mathrm{T}} \cdot \vec{x}^{*}
$$

where $a$ is a unit vector normal to the limit state surface and directed toward $G<g_{i}$, and $x^{*}$ is the design point. The first-order approximation of the failure probability can be obtained as

$$
p\left(G \leq g_{i}\right)=\Phi(-\beta)
$$

where $\Phi(\cdot)$ is the standard normal cumulative probability operator [30]. This approximation is accurate if the limit state surface is nearly flat in the neighborhood of the design point.

The design point is determined by solving an optimization problem that minimizes the distance from the origin in the reduced normal space to the limit state surface. A Newton-Raphson-type recursive algorithm, proposed by Rackwitz and Fiessler [31], as described in Equation (8), is implemented here:

$$
\vec{x}_{k+1}^{\prime}=\frac{1}{\left|\nabla G\left(\vec{x}_{k}^{\prime}\right)\right|^{2}}\left[\nabla G\left(\vec{x}_{k}^{\prime}\right)^{\mathrm{t}} \vec{x}_{k}^{\prime}-G\left(\vec{x}_{k}^{\prime}\right)\right] \nabla G\left(\vec{x}_{k}^{\prime}\right)
$$

where $k$ denotes the iteration number, and $\nabla G\left(\vec{x}_{k}\right)$ represents the gradient vector of the performance function at $\vec{x}_{k}^{\prime}$. The Rackwitz-Fiessler method linearizes the performance function at each iteration point, and uses the derivatives to find the next iteration point. The entire CDF can be constructed by repeating this FORM procedure to estimate the probability of the calculated permeability is smaller than a series of $g_{i}$ values in Equation (8).

In many applications, FORM requires only a small number of iterations for convergence. When the performance function is in implicit or numerical form, however, extra effort, for example, a finite difference scheme (Equation 10), may be required to derive the gradient of the performance function.

$$
\frac{\partial G(\vec{x})}{\partial x_{i}}=\frac{G\left(x_{i}+\Delta x_{i}\right)-G\left(x_{i}-\Delta x_{i}\right)}{2 \Delta x_{i}}
$$

Here, the step, $\Delta x_{i}$, is chosen as a small fraction of the standard deviation of random variables. Thus, the number of function evaluations required by each iteration of FORM will be $2 n+1$, where $n$ is the number of random variables.

\subsection{Proposed algorithm}

Since porosity data are widely available for many soil types and it can be accurately and routinely determined in laboratories, porosity statistics are generally easier to obtain than statistics for porous media permeability. An underlying assumption of this work is that the porosity of the simulated domain is a random variable.

Figure 2 presents a summary flowchart describing the proposed algorithm, as detailed below. First, an initial value for porosity is generated based on the probability distribution of the random variable porosity; it is then combined with a given particle size distribution to generate a random porous medium. LBM is then implemented to estimate the permeability of the generated domain. Based on the particular porosity and the particle size distribution, the resulting micropore configuration is subject to uncertainty. With sufficiently large numbers of samples, however, the average permeability of randomly generated porous media will approach a constant value dependent only on the porosity and the particle size distribution [32]. The simulation is considered converged if the relative error of the average permeability corresponding to the value derived from the previous number of samples is consistently less than $1 \%$ for five consecutive iterations. In this study, we found at least 25 samples were required to achieve a stable average permeability. Upon determination of an average permeability, the Rackwitz-Fiessler formula is employed to calculate the probability that the average permeability of the randomly generated porous media is smaller than a $g_{i}$. As illustrated in Figure 2, a particle size distribution is employed, but it is utilized to generate porous medium configurations as part of the performance function. Porosity is thus the only random variable for FORM input. 


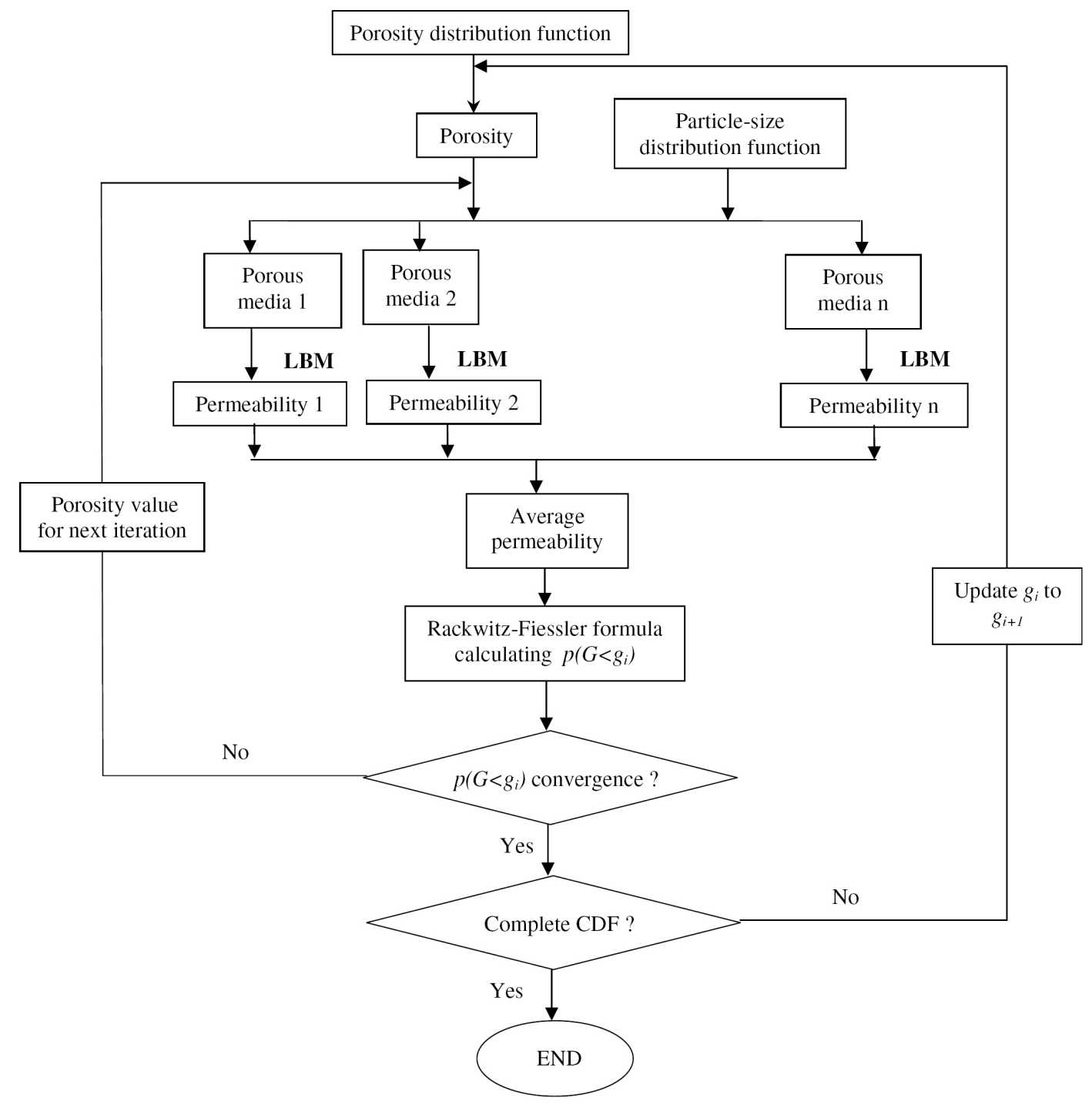

Figure 2. Flow chart for the LBM FORM algorithm.

In this situation, the Rackwitz-Fiessler iteration scheme [31] becomes a one dimensional Newton formula as expressed in Equation (11):

$$
x_{k+1}^{\prime}=\frac{1}{\frac{\partial G\left(x_{k}^{\prime}\right)}{\partial x}}\left[\frac{\partial G\left(x_{k}^{\prime}\right)}{\partial x} x_{k}^{\prime}-G\left(x_{k}^{\prime}\right)\right]
$$

Points on the CDF curve corresponding to $g_{i}$ are generated when the Rackwitz-Fiessler iteration scheme achieves convergence. Repeating this procedure for a series of $g_{i}$ values enables the construction of the entire CDF.

\section{Illustrative examples}

\subsection{Example model domains}

Simulations included use of randomly generated porous media with domain size $1 \mathrm{~mm} \times 1 \mathrm{~mm}$, and geo- metric mean particle diameters of 25,50 , and $100 \mu \mathrm{m}$, depicting particle sizes representative of very coarse silt to very fine sand. The particle size distribution employs a modified lognormal distribution, using a 95\% confidence interval for particle size to eliminate extreme values. The influence of particle sorting characteristics on permeability was also examined by varying the coefficient of variance (COV) of the particle diameters, i.e., COV of $0.01,0.31$ and 0.66 , which correspond to very well sorted, moderately sorted, and poorly sorted sediments. Summaries of the particle size distributions for simulated domains are listed in Table 1, while illustrations of the domains are presented in Figure 1.

\subsection{Evaluations of the LBM model}

A series of numerical simulations were conducted to evaluate LBM accuracy, its ability to estimate permeability, and numerical resolution requirements for the 
Table 1. Particle size distribution parameters employed in this work

\begin{tabular}{llllc}
\hline $\begin{array}{l}\text { Geometric mean } \\
\text { diameter }(\mu \mathrm{m})\end{array}$ & COV & $R_{\max } / R_{\min }$ Sorting & $\begin{array}{c}\text { Lattice } \\
\text { size }\end{array}$ \\
\hline 25 & 0.01 & 1.04 & Very well sorted & $800 \times 800$ \\
50 & 0.01 & 1.04 & Very well sorted & $400 \times 400$ \\
& 0.31 & 3.24 & Moderately sorted & $400 \times 400$ \\
& 0.66 & 10.51 & Poorly sorted & $540 \times 540$ \\
100 & 0.01 & 1.04 & Very well sorted & $300 \times 300$ \\
\hline
\end{tabular}

aforementioned example domains. Poiseuille flow was first simulated to test the accuracy of the LBM model. Numerical experiments with different channel widths were performed while holding the relaxation time, $\tau$, and Reynolds number, $R_{e^{\prime}}$ constant. Relative errors for the whole channel are calculated as

$$
\varepsilon=\frac{1}{N} \sqrt{\sum_{i=1}^{N}\left(u_{i}^{(n)}-u_{i}^{(a)}\right)^{2}}
$$

where $N$ is the number of lattice nodes along the channel width, and $u_{i}^{(n)}$ and $u_{i}^{(a)}$ are the numerical and analytical solutions, respectively. The relative error and the channel width present a linear relationship with a slope of -2.1 on a log graph, which indicates a second-order convergence of this LBM model in the spatial discretization, as described elsewhere $[17,26,33]$. The influence of $\tau$ on the accuracy of the LBM model was verified by numerical experiments employing different $\tau$ values from 0.6 to 1.2 with a step size of 0.2 , which provide a local minimum of the relative error at $\tau=0.8$.

To investigate the ability of LBM to accurately estimate permeability, LBM was employed with the randomly generated porous media under varying pressure gradient conditions. A linear relationship between pressure gradient and specific rate is identified with a low Reynolds number, i.e., $R_{e}<0.01$, which is consistent with applicable regions for Darcy's law $[16,34]$. In this study, $R_{e}$ is restricted to values less than 0.01 , and permeability is estimated as the ratio of flow rate and pressure gradient.

Effects of spatial discretization on permeability estimation for randomly generated porous media were investigated by varying the density of numerical grids on simulation domains. Results indicate that estimated permeability converges to a stable value with increasing spatial resolution, as stated elsewhere $[17,33]$. For COV of 0.01 and 0.31 , the number of grids per mean particle diameter, $m$, should be greater than 20 to achieve convergence on $k_{\mathrm{s}^{\prime}}$ while $m$ should be greater than 27 to achieve convergence for COV of 0.66. Specific lattice sizes employed in LBM simulations are listed in Table 1.

\section{Numerical results}

\subsection{Permeability statistics}

For the purpose of simplicity, a normal distribution with mean of 0.5 and COV of 0.12 is assumed for the random variable porosity. Under this condition, the probability of a negative porosity is as small as $1.5 \mathrm{E}-5$. A summary of statistical properties of the derived CDFs for the sample domains is presented in Table 2. The influence of particle size distributions on permeability CDFs are further illustrated in Figure 3 and Figure 4.

First, it is observed that domains with larger particle mean diameters or higher particle diameter COVs possess larger mean permeability values and higher probabilities of achieving larger permeability values. This phenomenon can be explained in terms of specific surface area, or the ratio of particle surface area in contact with fluid per unit volume. Specific surface areas are larger for the domains, which are well sorted or possess smaller particle mean diameters. Higher specific surface areas suggest greater surface area in contact with fluid, causing increased frictional resistance to fluid flow, thus leading to a reduction in permeability.

Second, it is shown in Table 2 that permeability COVs of all domains generally lie in the range of 1.0-1.5, which is about 10 times larger than the porosity COV of 0.12 . This suggests that permeability is subjected to greater uncertainty than porosity. In addition, permeability $\mathrm{COVs}$ of domains with mean diameter $50 \mu \mathrm{m}$ range from 1.0 to 1.3 , although the particle diameter COVs change 66 times from 0.01 to 0.66 . This indicates that, although the particle diameter COV will influence the permeability mean value, it will not directly affect the uncertainty of permeability. We believe it is the uncertainty of

Table 2. Statistical properties of derived permeability CDFs

\begin{tabular}{|c|c|c|c|c|c|c|}
\hline $\begin{array}{l}\text { Geometric mean } \\
\text { diameter }(\mu \mathrm{m})\end{array}$ & $\begin{array}{l}\text { Diameter } \\
\text { COV }\end{array}$ & $\begin{array}{l}\text { Permeability } \\
\text { mean (Darcy) }\end{array}$ & $\begin{array}{c}\text { Permeability } \\
\text { COV }\end{array}$ & \multicolumn{3}{|c|}{ Chi-square test significance level } \\
\hline 25 & 0.01 & 1.54 & 1.36 & 0.04 & 0.001 & 0.11 \\
\hline \multirow[t]{3}{*}{50} & 0.01 & 6.22 & 1.30 & 0.007 & $1 \mathrm{E}-05$ & 0.015 \\
\hline & 0.31 & 8.71 & 1.10 & 0.002 & $2 \mathrm{E}-06$ & 0.002 \\
\hline & 0.66 & 12.11 & 1.00 & 0.02 & $1 \mathrm{E}-04$ & 0.16 \\
\hline
\end{tabular}




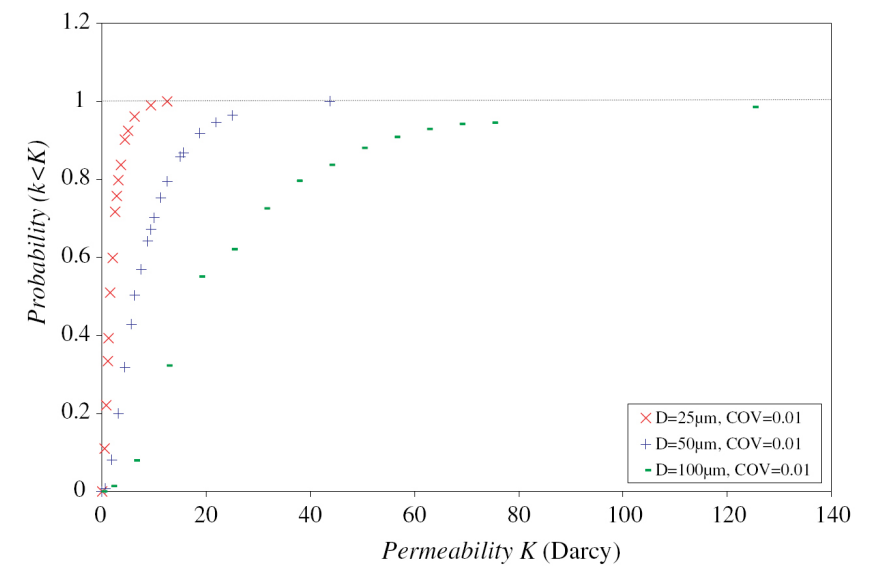

Figure 3. Influence of particle mean diameter on porous media permeability $\mathrm{CDF}$ for domains with geometric mean particle diameter $D=50 \mu \mathrm{m}$ and differing COV.

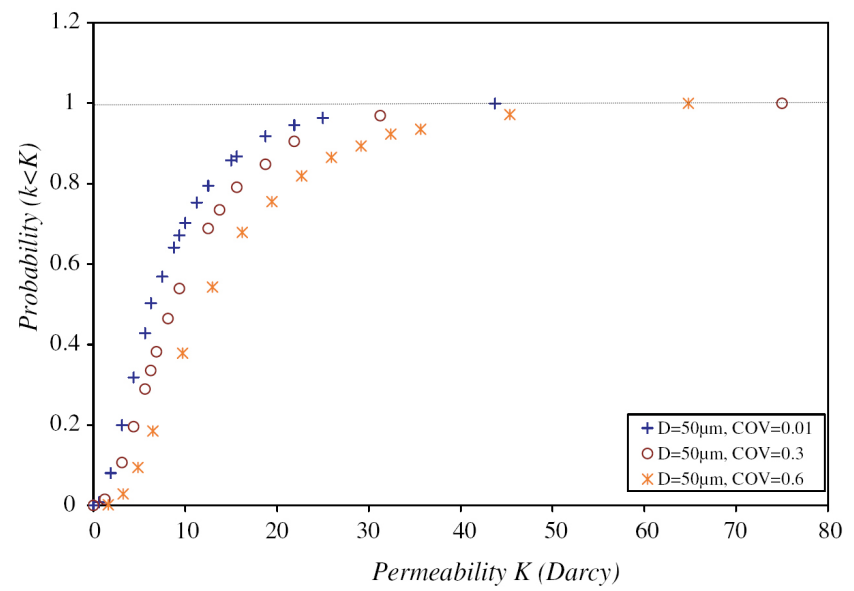

Figure 4. Influence of particle sorting on porous media permeability CDF for domains with geometric mean particle diameter $D=50 \mu \mathrm{m}$ and differing COV.

micropore structure configurations derived from porosity and particle size distributions that actually lead to the larger uncertainties in permeability.

\subsection{Applicability of permeability distributions}

Chi-square tests were applied to the derived permeability CDFs based upon normal, lognormal, and gamma distributions. The chi-square test significance levels are listed in Table 2, where a smaller significance level suggests that the model result is less significantly different from the given probability function, representing a better model fit. In this work, a lognormal distribution appears to provide the lowest significance level for all five modeling domains.

Further exploration of the ability of normal, lognormal, and gamma distributions to describe permeability is provided in Figure 5, which illustrates correspond- ing CDFs utilizing LBM FORM derived mean and COV. It is clear that normal distributions fail to represent the LBM FORM results at low probability, which can be attributed to normal distributions' allowance for the negative permeability values at extremely low probability. Although the Gamma distribution is limited to only positive values of permeability, it appears to overestimate the probability for the lower permeability. The failure of the Gamma distribution is likely associated with the constant nature of $\mathrm{COV}$, i.e., the Gamma distribution $\mathrm{COV}=1 / \sqrt{2}$, regardless of the mean value, which is incapable of fully describing the high uncertainty of porous media permeability. In this study, the lognormal distribution performs very well in describing the FORM-derived permeability CDF both at low and high probability regions, which are actually implied from two important characteristics of the lognormal distribution (i) exclusion of negative values and (ii) high right skewness. The applicability of the lognormal distribution to permeability in this study also agrees well with Woodbury and Sudicky [9], who evaluated more than 1000 samples for the Borden aquifer, suggesting that the lognormal distribution can be employed to describe the permeability distribution.

\subsection{Comparison with Monte Carlo simulations}

Monte Carlo simulation is a useful tool capable of addressing stochastic problems when only a basic working knowledge of probability and statistics is available. Given sufficient simulations, the Monte Carlo method can provide accurate simulation results in a simple but computationally demanding manner [30]. Evaluation of the necessary number of simulations required to guarantee the accuracy is thus critical in the proper employment of the Monte Carlo method. As opposed to common approaches that establish the number of model runs based upon experience or simplified tests, this study determined the required number of simulations by relating it to the relative error and probability based on equation [30],

$$
\varepsilon \%=\sqrt{\frac{(1-p)}{N \times p}} \times 200 \%
$$

where $\varepsilon$ is the error, $p$ is probability and $N$ is the number of simulations required. This equation was derived by considering the number of failures in $N$ trials as a binomial distribution, then approximating the binomial distribution with a normal distribution, and estimating the $95 \%$ confidence interval of the estimated probability of failure.

For the purpose of validation, Monte Carlo simulations were implemented on the domain with a particle mean diameter of $25 \mu \mathrm{m}$ and $\mathrm{COV}=0.01$. Equation (13) indicates that, for $p=0.1$, at least 14,400 Monte 

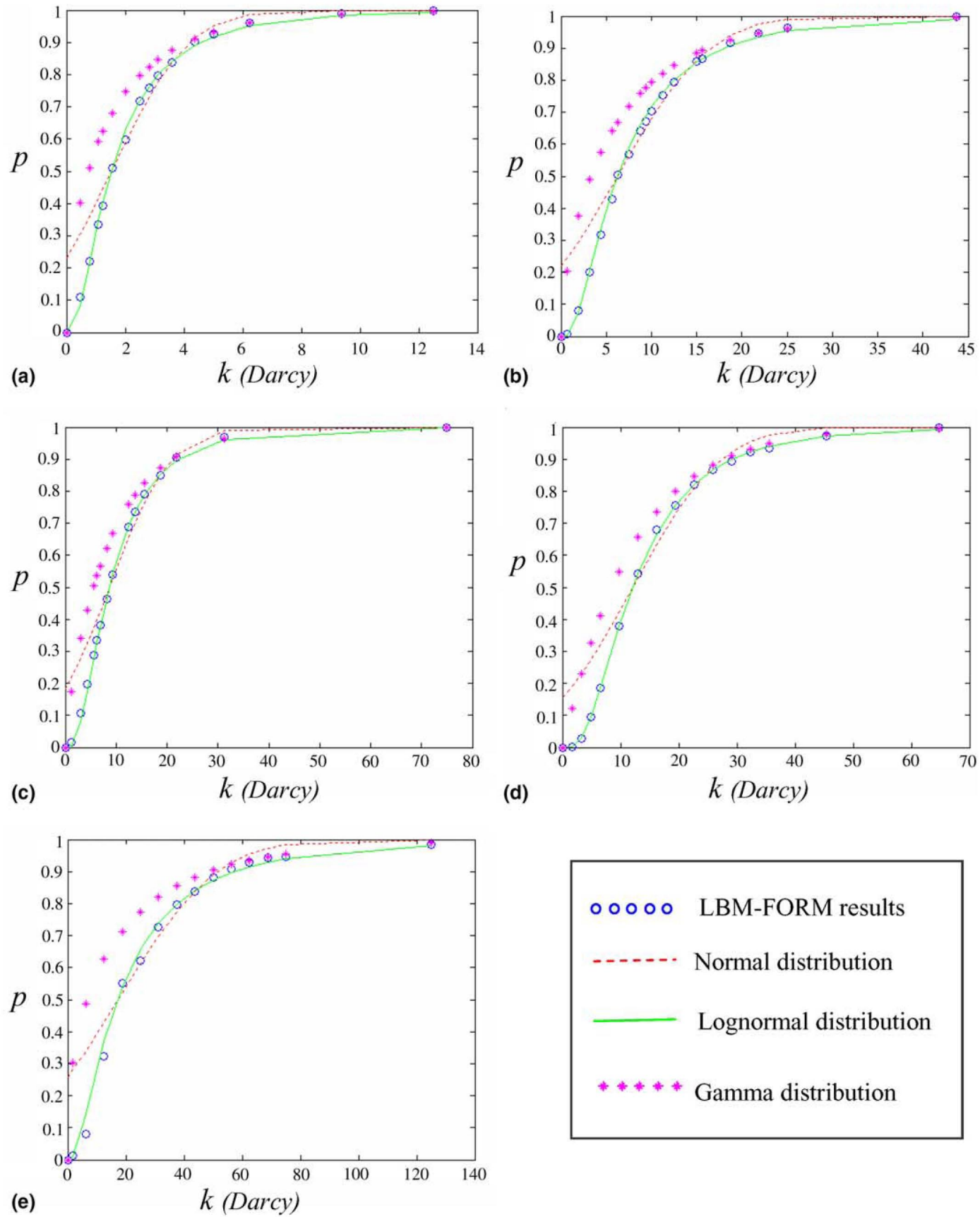

Figure 5. Comparison of FORM-derived permeability CDF with most commonly used Normal distribution CDF, Gamma distribution CDF, and Lognormal distribution CDF on the simulation domains with differing geometric mean particle diameter and COV. (a) $D=25 \mu \mathrm{m}, \mathrm{COV}=0.01$; (b) $D=50 \mu \mathrm{m}, \mathrm{COV}=0.01$; (c) $D=50 \mu \mathrm{m}, \mathrm{COV}=0.3$; (d) $D=50 \mu \mathrm{m}, \mathrm{COV}=0.6$; (e) $D=100 \mu \mathrm{m}$, $\mathrm{COV}=0.01$.

Carlo runs are required to achieve an error less than $5 \%$. As discussed in Section 2.4, the uncertainty of micropore structures for a given porosity and particle size distribution necessitates at least 25 simulations of different configurations to achieve a stable average permeability. 360,000 LBM simulations will thus be required to construct a CDF for $p>0.1$. In this study, the computing time required for LBM modeling of permeability depended on permeability values, varying from 0.5 to $10 \mathrm{~h}$ on a Dell Precision 650 Workstation; with lon- ger convergence times associated with higher permeability domains or denser numerical discretization resolutions. It is thus not feasible to perform all 360,000 LBM simulations due to computational limitations. Our approach to overcome this problem included (i) performing a smaller number of simulations based on available computing resources and (ii) quantitatively defining the relative error of Monte Carlo results based on Equation (12), which serves as a basis to evaluate the relative accuracy of LBM FORM results. 


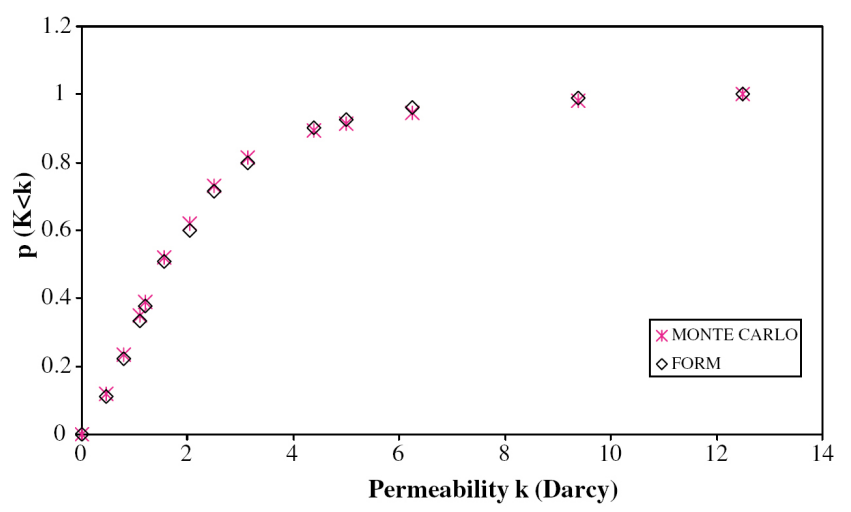

Figure 6. A comparison of Monte Carlo simulation results and FORM results for the simulation domain with particle mean diameter $D=25 \mu \mathrm{m}$ and $\mathrm{COV}=0.01$.

One thousand and six hundred Monte Carlo runs were computed, corresponding to 40,000 LBM simulations. Constructed CDFs are plotted in Figure 6 and compared well with FORM results. When the probability exceeds 0.4 , the largest relative error between the two methods is approximately $3.4 \%$. The largest relative error for the entire $\mathrm{CDF}$ is $8.5 \%$, occurring at the point $p=0.12$, the smallest probability point simulated. Based on Equation (12), 1600 Monte Carlo runs will provide an error of less than $6 \%$ for probabilities larger than 0.4 , and an error of $13.5 \%$ for $p=0.12$. LBM FORM results are thus within the relative error range of the Monte Carlo method, suggesting the relatively high accuracy of this method.

The FORM method employed in this work can achieve convergence within 2-6 iterations. Each iteration involves three function evaluations to calculate average permeability and gradient values. Although 15 points were used to construct the CDF employed in this work, 10 points are usually sufficient to generate a CDF [30]. Assuming an average of four FORM iterations to achieve convergence, three function evaluations for calculating average permeability and its gradient values, and 10 points on a CDF, approximately 120 averaged permeability values must be computed to construct a CDF through FORM. This is approximately $1 / 13$ times the 1600 Monte Carlo simulations used in this study, and approximately $1 / 120$ times the required 14,400 Monte Carlo simulations needed to construct a CDF possessing an error less than $5 \%$ when $p>0.1$, indicating the relatively high efficiency of LBM FORM relative to Monte Carlo simulations.

\section{Summary and conclusion}

Permeability, as a function of particle size distribution, porosity, and packing, is often the greatest source of uncertainty in simulating fate and transport of contaminants in the subsurface environment. Although permea- bility has previously been assumed as a random variable in groundwater modeling, the restriction on computational resources and the difficulty in relating microscale and macroscale uncertainties have resulted in reduced efforts to construct probability density functions for permeability. In this study, we proposed a new approach, LBM FORM, based on more easily derived porosity statistics and particle size distribution, to construct permeability CDFs through the combination of LBM and FORM.

LBM FORM was implemented to construct permeability CDFs of five randomly generated porous media; each possessing different particle size distributions. Results show that the domains with larger mean particle diameter or higher particle diameter COV tend to possess a higher probability of achieving larger permeability. Permeability values are subjected to higher uncertainty than the porosity and particle diameters because of the uncertainty of the micropore structure configurations. Lognormal distributions modeled well the permeability CDF constructed for a variety of domains examined in this study. Accuracy of the proposed method was confirmed by comparison with Monte Carlo simulations for one example simulation domain. The largest relative error is approximately $3.4 \%$ when the probability exceeds 0.4 , and is $8.5 \%$ when probability is less than 0.4 , both of which are within the relative error associated with the Monte Carlo method. Further, this work demonstrated that the Monte Carlo method is severely limited by computational requirements, making it extremely difficult to accurately construct an entire permeability CDF curve by Monte Carlo; LBM FORM, however, was found to be approximately 13-120 times more efficient than traditional Monte Carlo simulations.

The primary contribution of this effort derives from the development of a new approach to calculate permeability CDFs by combining LBM and FORM. Although it provides higher accuracy and efficiency than MonteCarlo simulations, it is worthy to note several directions for enhancements of the method. First, the LBM method implemented in this study is in the BGK form [35] with a linear collision operator. The accuracy of permeability based on BGK LBM is dependent on the fluid viscosity and thus on the relaxation time. We chose an optimized value of the relaxation time $\tau(\tau=0.8)$ to eliminate this dependency. In the future, we suggest the use of more sophisticated LBM schemes to simulate fluid flow in porous media. For example, a two relaxation time (TRT) LBM $[36,37]$ will be able to annihilate the permeability dependence on the viscosity with a specific choice of the free eigenvalues. Further, the convergence rate is accelerated when using higher viscosity values for a TRT LBM. A second potential enhancement of the method is associated with the reliability method employed. While FORM performed well in example domains in this study, more advanced methods, such as SORM or 
other modified forms of FORM, might be required for situations that are more complicated. Finally, the simulation results in this work are based on randomly generated, two-dimensional simplified porous media. Future modeling efforts will benefit from use of more sophisticated porous media packing modules to more closely reflect actual field situations. Applications of the proposed framework with more sophisticated LBM and reliability methods for three-dimensional porous media should greatly assist future researchers in advancing fundamental understanding of the primary factors influencing permeability within porous media.

\section{Acknowledgments}

This material is based upon work supported by the National Science Foundation under Grant No. 0088912. Any opinions, findings, and conclusions or recommendations expressed in this material are those of the authors and do not necessarily reflect the views of the National Science Foundation. We also thank three anonymous reviewers from Advances in Water Resources for their helpful comments.

\section{References}

1. A. E. Scheidegger, The physics of flow through porous media, University of Toronto Press, Buffalo (1974).

2. M. Sahimi, Flow and transport in porous media and fractured rock: from classical methods to modern approaches, VCH, Weinheim (1995).

3. Z. Lu and D. Zhang, On importance sampling Monte Carlo approach to uncertainty analysis for flow and transport in porous media, Adv Water Resour 26 (2003), pp. 1177-1188.

4. N. Sitar, J. D. Cawlfield, and A. D. Kiureghian, First-order reliability approach to stochastic analysis of subsurface flow and contaminant transport, Water Resour Res 23 (1987), pp. 794-804.

5. M. M. Hamed, P. B. Bedient, and J. P. Conte, Numerical stochastic analysis of groundwater contaminant transport and plum containment, J Contam Hydrol 24 (1996), pp. 1-24.

6. T. H. Skaggs and D. A. Barry, The first-order reliability method of predicting cumulative mass flux in heterogeneous porous formations, Water Resour Res 33 (1997), pp. 1485-1494.

7. P. R. Johnston, Revisiting the most probable pore-size distribution in filter media: the gamma distribution, Filtr Sep 35 (1998), pp. 287-292.

8. R. A. Cooke, S. Mostaghimi, and F. Woeste, Effect of hydraulic conductivity probability distribution function on simulated solute leaching, Water Env Res 67 (1995), pp. 159-168.

9. A. D. Woodbury and E. A. Sudicky, The geostatistical characteristics of the Borden aquifer, Water Resour Res 27 (1991), pp. 533-546.

10. M. Turcke and B. Kueper, Geostatistical analysis of the Borden aquifer hydraulic conductivity, J Hydrol 178 (1996), pp. 223-240.

11. P. Kennedy and A. Woodbury, Geostatistics and Bayesian updating for transmissivity estimation in a multiaquifer system in Manitoba, Canada, Ground Water 40 (2002), pp. 273-283.

12. M. M. Hamed, P. B. Bedient, and C. N. Dawson, Probabilistic modeling of aquifer heterogeneity using reliability methods, Adv Water Resour 19 (1996), pp. 277-295.

13. T. H. Skaggs and D. A. Barry, Assessing uncertainty in subsurface solute transport: efficient first-order reliability methods, Env Software 11 (1996), pp. 179-184.

14. R. Portielje, T. Hvitved-Jacobsen, and K. Schaarup-Jensen, Risk analysis using stochastic reliability methods applied to two cases of deterministic water quality models, Water Res 34 (2000), pp. 153-170.

15. H. R. Maier, B. J. Lence, B. A. Tolson, and R. O. Foschi, First-order reliability method for estimating reliability, vulnerability, and resilience, Water Resour Res 37 (2001), pp. 779-790.

16. D. H. Rothman, Cellular-automaton fluids: a model for flow in porous media, Geophysics 53 (1988), pp. 509-518.

17. C. Pan, M. Hilpert, and C. T. Miller, Pore-scale modeling of saturated permeabilities in random sphere packings, Phys Rev E 64 (2001), pp. 0066702-1-0066702-9.

18. C. Manwart, U. Aaltosalmi, A. Koponen, R. Hilfer, and J. Timonen, Lattice-Boltzmann and finite-difference simulations for the permeability for three-dimensional porous media, Phys Rev E 66 (2002), pp. 1-11.

19. Y. Keehm, T. Mukerji, and A. Nur, Permeability prediction from thin sections: 3D reconstruction and lattice-Boltzmann flow simulation, Geophys Res Lett 31 (2004) Art. No. L04606.

20. D. Zhang, R. Zhang, S. Chen, and W. E. Soll, Pore scale study of flow in porous media: scale dependency, REV, and statistical REV, Geophys Res Lett 27 (2000), pp. 1195-1198.

21. J. Eggleston and S. Rojstaczer, The value of grain-size hydraulic conductivity estimation: comparison with high resolution insitu field hydraulic conductivity, Geophys Res Lett 28 (2001), pp. 4255-4258.

22. A. Lerman, Geochemical processes: water and sediment environments, Wiley, New York (1979).

23. M. D. Fredlund, D. G. Fredlund, and G. W. Wilson, An equation to represent grain-size distribution, Can Geotech J 37 (2000), pp. 817-827.

24. A. Yang, C. T. Miller, and L. D. Turcoliver, Simulation of correlated and uncorrelated packing of random size spheres, Phys Rev E 53 (1996), pp. 1516-1524.

25. S. Chen and G. D. Doolen, Lattice Boltzmann method for fluid flow, Ann Rev Fluid Mech 30 (1998), pp. 329-364.

26. D. A. Wolf-Gladrow, Lattice-gas cellular automata and lattice Boltzmann models: an introduction, Lecture notes in mathematics, Springer, New York (2000).

27. A. Cancelliere, C. Chang, E. Foti, D. H. Rothman, and S. Succi, The permeability of a random medium: comparison of simulation with theory, Phys Fluids A, Fluid Dyn 12 (1990), pp. 2085-2088.

28. A. W. J. Heijs and C. P. Lowe, Numerical evaluation of the permeability and Kozeny constant for two types of porous media, Phys Rev E 51 (1995), pp. 4346-4352.

29. H. Chen, S. Chen, and W. H. Matthaeus, Recovery of the NavierStokes equations using a lattice-gas Boltzmann method, Phys Rev E 45 (1992), pp. R5339-R5342.

30. A. Haldar and S. Mahadevan, Probability, reliability, and statistical methods in engineering design, John Wiley, New York (2000).

31. R. Rackwitz and B. Fiessler, Structural reliability under combined random loading sequences, Comput Struct 9 (1978), pp. 484-494.

32. A. Koponen, M. Kataja, and J. Timonen, Permeability and effective porosity of porous media, Phys Rev E 56 (1997), pp. 3319-3325.

33. R. S. Maier, D. M. Kroll, Y. E. Kutsovsky, H. T. Davis, and R. S. Bernard, Simulation of flow through bead packs using the lattice Boltzmann method, Phys Fluids 10 (1998), pp. 60-74.

34. J. Bear, Dynamics of fluids in porous media, Dover Publications, New York (1972).

35. P. Bhatnagar, E. P. Bross, and M. K. Krook, A model for collision processes in gases. I. Small amplitude processes in charged and neutral one-component systems, Phys Rev 94 (1954), pp. 511-525.

36. D. d'Humieres, I. Ginzburg, M. Krafczyk, P. Lallemand, and L. S. Luo, Multiple-relaxation-time lattice Boltzmann models in three dimensions, Phil Trans Roy Soc Lond Ser A-Math Phys Eng Sci 360 (2002), pp. 437-451.

37. P. Lallemand, D. d'Humieres, L.-S. Luo, and R. Rubinstein, Theory of the lattice Boltzmann method: three-dimensional model for linear viscoelastic fluids, Phys Rev E 67 (2003) Art. No. 021203, Part 1. 